\title{
The NuTel Telescope for Observing Very High Energy Tau Neutrinos from AGN
}

\author{
Ping Yeh*, Koji Ueno, Yankun Chi, Yuri Velikzhanin, Min-Zu Wang, Jing-Ge Shiu and Ching-Cheng Hsu \\ Department of Physics, National Taiwan University, Taipei, TAIWAN 106 \\ ${ }^{*}$ Member, IEEE
}

\begin{abstract}
The NuTel collaboration is building a wide field-ofview Čerenkov telescope on a mountain to observe air showers generated by tau neutrinos emerging from another mountain. Such a technique will be realized for the first time in the $\nu_{\tau}$ energy range of $1 \mathrm{PeV}$ to $1000 \mathrm{PeV}$. We describe the design of the optical system, electronics and data acquisition system of the telescope in this paper.
\end{abstract}

Index Terms-Active galactic nuclei, Earth skimming, tau neutrino appearance, extensive air shower, Čerenkov telescope.

\section{INTRODUCTION}

$\mathbf{N}$ EUTRINOS play important roles in several branches of sciences: particle physics (neutrino oscillation), astroparticle physics ( $\mathrm{TeV}$ gamma rays and ultra-high energy cosmic rays), and cosmology (energy density of the universe).

The origin of ultra-high energy cosmic rays (UHECR) is still a great puzzle [1]. Bottom-up theories propose that they originate from energetic processes in Active Galactic Nuclei (AGN) or Gamma Ray Bursts (GRB). The hadron component of such energetic processes could interact with accreting materials to produce neutrinos through the decay of charged pions. On the other hand, top-down theories like to suggest that UHECR are decay products of topological defects or heavy relic particles. According to these theories, there are more neutrinos than gamma rays and protons [2]. Measurement of the neutrino flux at and below the ankle region (i.e. $<3 \times 10^{18} \mathrm{eV}$ ) provides a good discriminator between the two scenarios.

Studies of small scale anisotropy of cosmic rays near the ankle region reveal a small but significant excess of events near galactic center (GC) [3], [4], [5], [6], where a supermassive black hole with mass $2.6 \times 10^{6} M_{\odot}$ is known to exist [7]. These anisotropies could be signals of potential source or acceleration site of cosmic rays in galactic center. The neutrino flux from $\mathrm{GC}$ would be higher than prediction if it is a point source of neutrinos [8].

Unlike charged cosmic rays, neutrinos do not bend in magnetic field and provide a pointing capability to the production site. Compared to photons which suffer attenuation during propagation, the weakly-interacting neutrinos are complementary probes to the universe. However, the difficulty of neutrino observation has always been the weak interaction which directly translates to rare signals.

Recent results on atmospheric neutrinos add an interesting twist to cosmological neutrino detection. Super-Kamiokande data strongly suggest that mиоn neutrinos oscillate into tau neutrinos [9]. Since cosmic neutrinos are predominantly produced via pion decays, one does not expect much directly produced cosmic $\nu_{\tau}$ flux [10]. However, with the maximum oscillation seemingly taking place, the fluxes $\mathcal{F}\left(\nu_{\mu}\right): \mathcal{F}\left(\nu_{\tau}\right)$ is expected to reach $1: 1$ for distant observers. Detecting a $\tau$ lepton descendent on Earth would not only probe AGN/GRB/GC mechanisms, but would also constitute a $\nu_{\tau}$-appearance experiment.

All neutrino experiments need a large target volume to convert incoming neutrions into detectable objects like charged leptons or proton recoil for observation. Traditional design is surrounding the target volume by detectors. The target has to be large enough to have sensitivity. Targets such as lake water (Baikal [11]), sea water (ANTARES [12], NESTOR [13]) and southpole ice (AMANDA [14], IceCube [15]) have been used. To shield the detectors from backgrounds from cosmic rays and other sources, such experiments are located deep under the Earth surface. Although the target is free, the vast amount of sensors surrounding it drives the cost high.

The idea of using Earth [16], [17] or mountains [18], [19] as target and use atmosphere as a calorimeter for shower development has been a new trend. AMANDA and Auger [20] are using horizontal showers to observe high energy neutrinos.

The NuTel collaboration [21] is specifically using the moutain idea to build a wide field-of-view Čerenkov telescope on a mountain to observe air showers emerging from another mountain or the Earth. The target mountain also function as a shield to block cosmic rays and star lights. Among all flavors of neutrinos, this technique is sensitive to $\nu_{\tau}$ because only the $\tau$ lepton has a reasonable probability to escape the mountain and initiate extensive air showers. A preliminary feasibility study showed that this technique performs best in the $\nu_{\tau}$ energy range of $1 \mathrm{PeV}$ to $1000 \mathrm{PeV}$ [22], [23], and the most plausible source in this energy range are AGNs [24], which produces an integral $\tau$ lepton flux approximately $7.5 \mathrm{~km}^{-2} \mathrm{sr}^{-1} \mathrm{yr}^{-1}$.

The candidate site for the observatory is Mt. Hualalai on the Big Island of Hawaii facing Mauna Loa with a valley of $30-$ $40 \mathrm{~km}$ wide. The galactic center passes the field of view several times a year.

In this paper we describe the design of the optical system, electronics and data acquisition system of the telescope. 


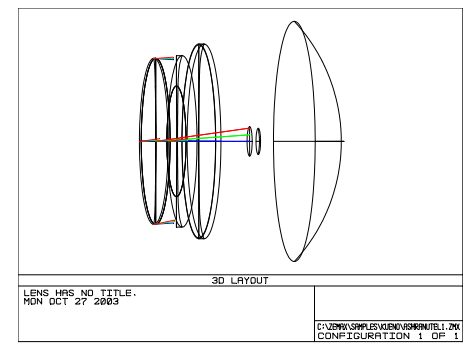

Fig. 1. Schematics of the optics of one telescope. The focal plane is a curved surface, and the MAPMTs are represented as the space between the focal surface and a larger surface behind it. Three rays of light are shown to be obstructed by the MAPMT.

\section{THE OptICS}

The sensitivity of the telescope increases with the aperture, while the cost increases even faster with the aperture. Instead of a big aperture telescope, we use identical telescopes with small aperture as building blocks. This design has very high flexibility: we can point 2 telescopes to the same field of view for coincidences to reject random background photons, or point some telescopes in different directions to form a larger total field of view.

The Cerenkov photons produced by particles in an extensive horizontal air shower typically arrive at an observation point with angular span of $1^{\circ}$ to $2^{\circ}$. An angular resolution of $0.5^{\circ}$ per pixel is sufficient to form a course-grained image of the shower. Such a resolution can be achieved with a low-cost optical system with multi-anode photomultiplier tubes (MAPMTs). The major background during observation is expected to be from random ambient photons. The night sky background (NSB) has been measured to be $\approx 2000$ photons $/ \mathrm{m}^{2} / \mathrm{sr} / \mathrm{ns}$ [25], [26]. Due to the randomness of the background, the signal/background discrimination will mainly come from the clustering of fired pixels.

The building block telescope has a spherical mirror to reflect Cerenkov photons onto a curved focal plane. Three corrector lens with the Baker-Nunn [27], [28] design are used to reduce aberrations, see figure 1 for the geometry. To reduce dead area among MAPMT pixels, an array of mini-lens light collectors will be used to direct lights from the focal plane to the surface of MAPMTs [29], [30], see figure 2 for a drawing. The same light collector has been proposed to the $\mathrm{LHCb}$ experiment in CERN. Table I lists the parameters of the optical system.

A preliminary measurement of the random background photon flux on the LuLin mountain in central Taiwan confirms that NSB is approximately 2000 photons $/ \mathrm{m}^{2} / \mathrm{sr} / \mathrm{ns}$ for wavelength in $300 \mathrm{~nm}-400 \mathrm{~nm}$, while the background from dark mountains is 10 times less. With the optical parameters listed in table I, the average photoelectron rate from NSB is $17 \mathrm{MHz}$ per pixel.

Air shower simulations reveal that the Cerenkov photons from a shower arrive at the telescope in bunches with a typical spread of arrival time around $10 \mathrm{~ns}$ to $100 \mathrm{~ns}$, depending on the

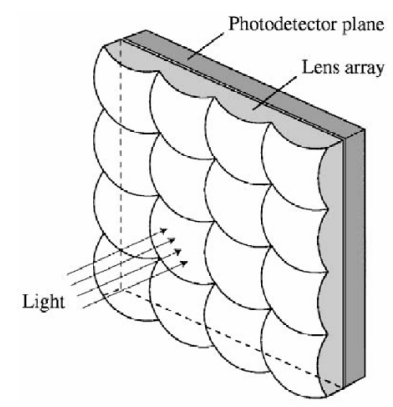

Fig. 2. The proposed light collector in front of MAPMT for LHCb.

TABLE I

PARAMETERS OF THE OPTICAL SYSTEM OF ONE TELESCOPE.

\begin{tabular}{|l|l|}
\hline $\begin{array}{l}\text { Mirror diameter } \\
\text { focal length }\end{array}$ & $1.2 \mathrm{~m}$ \\
Typical transportation efficiency & $0.6 \mathrm{~m}$ \\
\hline Field of View & $50 \%$ \\
\hline Number of MAPMTs & $\pm 4^{\circ}$ \\
\hline MAPMT pixel number & $2 \times 2$ \\
pixel size & $8 \times 8$ \\
quantum efficiency & $3 \mathrm{~mm} \times 3 \mathrm{~mm}$ \\
\hline Angular span per pixel & $0.5^{\circ} \times 0.5^{\circ}$ \\
\hline
\end{tabular}

distance between the telescope and the shower axis. At least one photoelectron per pixel can be measured within a range of $\approx 1 \mathrm{~km}$ for normal incident showers with $E>1 \mathrm{PeV}$.

\section{THE ElECtRonics}

Due to the high rate from NSB, a fast electronics is needed to get rid of the background. However, the cost must be low. So a design principle of using as many commodity as possible is chosen. That leads us to use low-cost amplifiers, 10-bit pipeline ADCs and low-cost FPGAs. Another feature of the design is that there is no analog discriminator. All trigger decisions are in digital space. We describe the design in this section.

We use Hamamatsu MAPMT model H7546 with $8 \times 8=64$ pixels as the photon sensor. The quantum efficiency reaches maximum for wavelength of $350-450 \mathrm{~nm}$, a good match to Čerenkov photons. Typical pulse width is 6 ns. The gain is around $3 \times 10^{5}$ at $800 \mathrm{~V}$, but not uniform for all pixels. The maximum gain can be 5 times the minimum gain.

The non-uniformity is reduced in 2 ways. First we connect tubes with similar maximum gain to the same channel of the high voltage power supply (HV). Second we adjust the gain of preamplifiers channel by channel.

We use Tico VHQ 202M dual-channel high voltage power supply for the MAPMTs. Four MAPMTs are connected to the same HV channel to reduce cost on HV. The HV power supply can be controlled with a VME module.

The amount of Čerenkov photons that a telescope receives is to a large extent proportional to the energy of primary $\tau$ lepton. The range of interest spans from $1 \mathrm{PeV}$ to hundreds of $\mathrm{PeV}$. That requires a dynamic range of $\approx 500$. With 6 to 10 counts for single photon to obtain a reasonable $\mathrm{S} / \mathrm{N}$ ratio, the 10-bit ADC alone can't reach the desired dynamic range. 
One cost-effective way of enlarging the dynamic range is via signal sharing. A 64-channel signal-sharing board is plugged onto the MAPMT anode pins. On this board every channel is connected to 2 other channels resistively to form a cross-talk network. The connected pixels are approximately $2.1^{\circ}$ away in field of view to reduce the probability of being shined by the same shower. The current division is 0.9:0.05:0.05. The $10 \%$ coupling to 2 other channels does not affect too much on signal, but when the signal pixel is saturated by large pulse, the signal-shared pixel can be used to compute the pulse height. With the signal-sharing board the preamplifier is linear from 1 photoelectron to 400 photoelectrons, a factor of 4 increase in dynamic range.

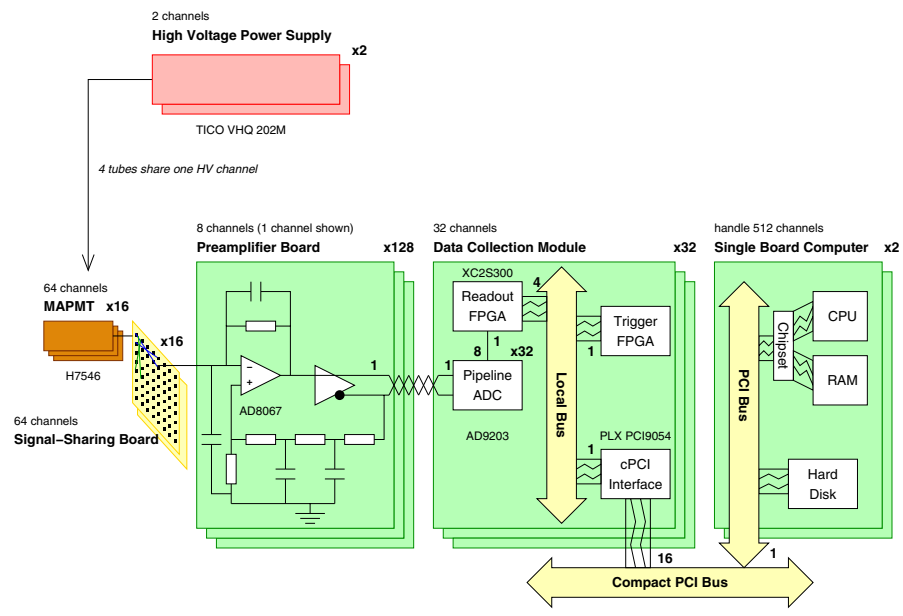

Fig. 3. The block diagram of the electronics.

A row of the signal-sharing board is connected to a chargesensitive preamplifier board for signal amplification and shaping. A charge collection capacitor at the preamplifier is discharged with a time constant of 387 ns. The choice of time constant will be explained later. With this relatively long time constant and the high background rate from NSB, it is foreseen that the shaped pulses will pile up. The output voltage will reach a DC level of $56 \mathrm{mV}$ for NSB. A feedback network as shown in figure 3 is being investigated for discharging the pileup voltage.

The shaped analog signals from preamplifiers are passed to the Data Collection Modules (DCM) via twisted pairs. Each DCM card accepts 32 analog inputs. One 10-bit pipeline ADC running at $40 \mathrm{MHz}$ digitizes the voltage of one input channel. Figure 3 shows the block diagram of the electronics.

The preamplifier time constant of $387 \mathrm{~ns}$ and the clock of $40 \mathrm{MHz}$ are matched to allow determination of number of photoelectrons at each clock interval with a simple double correlation logic in FPGA, i.e., $\exp (-25 / 387)=15 / 16$. Figure 4 shows this process. With such logic in place, the effective shaping time of the system is $25 \mathrm{~ns}$. This allows us to operate the telescope in a harsh background condition.

The Xilinx XC2S300 FPGA of Spatan-IIe family is used as the readout FPGA. It has 16 dual-port memory blocks of $256 \times 16$ bits each. Seven blocks are designated as cyclic

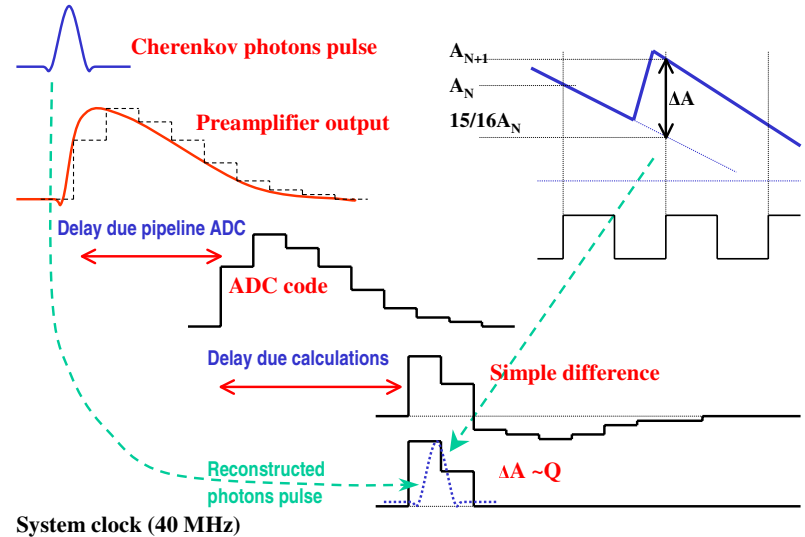

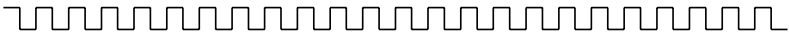

Fig. 4. Calculation of charge in every clock interval.

memories to store outputs from 8 ADCs. Nine blocks are used as buffers for triggered events, where each event contains 8 consecutive clocks of ADC counts for time analysis as well as a 60-bit time stamp, a 30-bit trigger number and the integrated background charge in a pre-configured time interval. The depth is 256 clocks for the cyclic memories and 32 events for the buffer, respectively. One out of 32 event buffer is reserved specifically for very high energy events. A buffer-full flag is raised when all 31 normal event buffers are occupied. Figure 5 shows the data layout in memory.
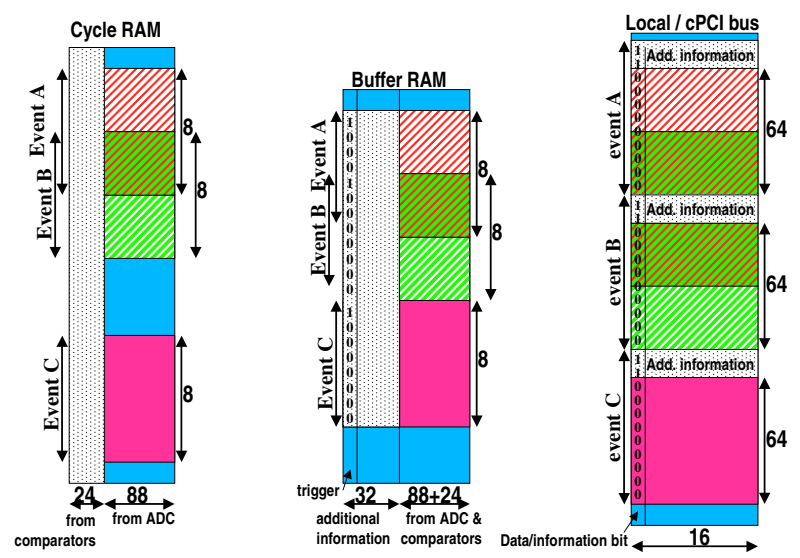

Fig. 5. Data layout of cyclic and buffer memories.

Calculated charge in each clock interval is passed to 3 programmable digital comparators in the readout FPGA (figure 6). All 3 thresholds (Low, High, Very High) are configurable. The events that passes VH threshold is treated specially that they are stored in the very high energy event buffer. The result of comparisons of 8 readout FPGAs are passed to the trigger FPGA for cluster trigger. The cluster trigger is also configurable.

The trigger FPGA on board DCM applies the trigger al- 


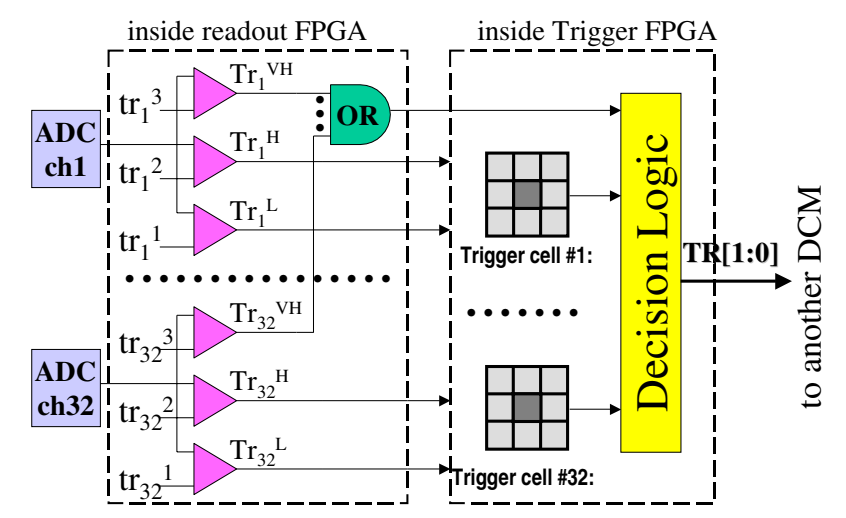

Fig. 6. On-board trigger logic

gorithm on the 32 channels local to the same DCM board to produce one trigger bit. The trigger bit is sent to the next DCM to be OR-ed with its trigger bit. This operation is repeated in a daisy chain fashion until the OR-ed trigger bit reaches the terminal DCM in the crate, otherwise known as the Master DCM. The Master DCM has identical hardware with all other DCMs but has a slightly different firmware in the FPGA. The daisy chain is pipelined so there is a 16 clock delay but zero deadtime.

Figure 7 shows the interconnection among DCMs. One of the Master DCMs can be configured to perform coincidence with the Master DCM in another crate. The coincidence can be disabled for background study. When the Master DCM finds the trigger bit is set, it notifies all DCMs in the same crate to store data into buffer by raising the trigger signal. The buffer will be read out and cleared by data acquisition system at an appropriate time. A buffer-full condition would block the Master DCM from raising trigger signals, but VHL events are not subject to this limitation.

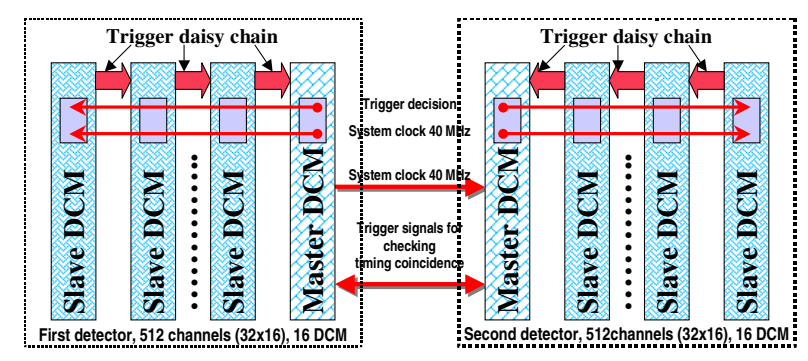

Fig. 7. Timing and trigger interconnections

The data acquisition system (DAQ) contains 2 compact PCI crates, each with one single board computer (SBC) and 16 DCM boards. One SBC collects event data from 16 DCMs in the same crate through the $33 \mathrm{MHz}$ 32-bit wide compact PCI bus. Due to the overhead of starting and terminating data transfer on the compact PCI bus, the event readout is performed only when the event buffer of DCMs reach halffull (16 events). Sixteen events from each DCM are read into one SBC, and assembled in the SBC's memory. The CPU on
SBC then performs more sophisticated background rejection algorithm to further reduce data volume. The data is finally gathered onto one SBC and written to storage.

On board the SBC a Linux ${ }^{1}$ operating system is used to coordinate resources and tasks. The multi-tasking capability and POSIX-compliant multi-thread libraries make it easy to execute event readout, trigger and event output tasks at the same time. The interrupt latency of the Linux operating system has been measured with LPT port. There is a fluctuation because Linux is not a real time system. However, the distribution concentrates on region of $0-10 \mu \mathrm{s}$. The maximum latency is found to be less than $20 \mu \mathrm{s}$, which is sufficient for the DAQ task. Full reconstruction of events is done also in SBC during day time when the telescopes and most electronics are turned off.

\section{Calibration and Field Test}

Besides ordinary calibration of the optics and electronics, the calibration of pointing is of special importance for discriminating mountain-coming against sky-coming air showers. A preliminary study showed that with the current design of the telescope we can readily observe $\mathrm{TeV}$ gamma rays from $\mathrm{Crab}$ nebula, which will be used as the lighthouse for calibration.

Currently we are building a 4-telescope prototype with 1024 channels in total. Instead of directly installing the prototype on Mt. Hualalai where no utility infrastructure exists, we'll use the AMiBA [31] site on Mauna Loa in year 2004. It is an important step toward understanding the detector and operation for future optimization for the performance for the full-scale detector.

\section{SUMmary}

The NuTel collaboration is building a neutrino telescope to observe Čerenkov photons from air showers produced by $\tau$ leptons emerging from a mountain or Earth surface. Such a shower is a distinct signature of $\operatorname{cosmic} \tau$ neutrinos, that makes NuTel a $\tau$-appearance neutrino experiment.

With emphasis on low cost and speed prototyping, commodity amplifiers and FPGAs have been used, as well as an open source operating system for SBC. A signal-sharing board is used to enlarge the dynamic range, and a feedback network is being investigated for eliminating the pileup. One major decision is that all triggers are done in digital part of the electronics, relying heavily on the FPGA firmwares. The prototype system will be installed on Mauna Loa of Hawaii Big Island for field test in 2004.

\section{ACKNOWLEDGMENT}

This work is funded by Ministry of Education of Taiwan under grant MOE 89-N-FA01-1-4-2.

\footnotetext{
${ }^{1}$ Linux is a trademark of Linus Torvalds.
} 


\section{REFERENCES}

[1] J. G. Learned and K. Mannheim, "High-energy neutrino astrophysics," Ann. Rev. Nucl. Part. Sci., vol. 50, pp. 679-749, 2000.

[2] P. Bhattacharjee and G. Sigl, "Origin and propagation of extremely high energy cosmic rays," Phys. Rept., vol. 327, pp. 109-247, 2000. e-print: astro-ph/9811011

[3] M. A. Huang, Ph.D. dissertation, University of Utah, 1996.

[4] D. J. Bird et al., "Study of broad-scale anisotropy of cosmic-ray arrival directions from $2 \times 10^{17}$ to $10^{20}$ electron volts from Fly's Eye data," Astrophys. J., vol. 511, p. 739, 1999.

[5] N. Hayashida et al., "The anisotropy of cosmic ray arrival directions around $10^{18}$ ev," Astropart. Phys., vol. 10, pp. 303-311, 1999. e-print: astro-ph/9807045

[6] J. A. Bellido, R. W. Clay, B. R. Dawson, and M. Johnston-Hollitt, "Southern hemisphere observations of a $10^{18}$ ev cosmic ray source near the direction of the galactic centre," Astropart. Phys., vol. 15, pp. 167175, 2001. e-print: astro-ph/0009039

[7] R. Schoedel et al., "A star in a 15.2-year orbit around the supermassive black hole at the centre of the Milky Way," Nature, vol. 419, p. 694, 2002.

[8] K. Takahashi and S. Nagataki, "Vhe neutrinos and gamma-rays as probes for anisotropy of arrival directions of eev cosmic rays," 2001. e-print: astro-ph/0108507

[9] S. Fukuda et al., "Tau neutrinos favored over sterile neutrinos in atmospheric muon neutrino oscillations," Phys. Rev. Lett., vol. 85, pp. 39994003, 2000. e-print: hep-ex/0009001

[10] H. Athar and G.-L. Lin, "Implications of $\bar{\nu}_{e} e^{-} \rightarrow W^{-} \gamma$ for high-energy $\bar{\nu}_{e}$ observation," Astropart. Phys., vol. 19, pp. 569-574, 2003. e-print: hep-ph/0108204

[11] C. Spiering et al., "The Baikal experiment," Nucl. Phys. Proc. Suppl., vol. 14B, pp. 51-59, 1990.

[12] L. Moscoso, "Neutrino telescopes under the ocean: The case for ANTARES," Nucl. Phys. Proc. Suppl., vol. 77, pp. 492-497, 1999. eprint: hep-ex/9809020

[13] F. Ameli et al., "Data acquisition electronics for NESTOR experiment: Project and tests," Nucl. Instrum. Meth., vol. A423, pp. 146-156, 1999.

[14] F. Halzen et al., "The AMANDA neutrino telescope," Nucl. Phys. Proc. Suppl., vol. 77, pp. 474-485, 1999. e-print: hep-ex/9809025

[15] J. Ahrens et al., "IceCube: The next generation neutrino telescope at the south pole," Nucl. Phys. Proc. Suppl., vol. 118, pp. 388-395, 2003. e-print: astro-ph/0209556

[16] G. Domokos and S. Kovesi-Domokos, "Observation of UHE neutrino interactions from outer space," 1997. e-print: hep-ph/9801362

[17] J. L. Feng, P. Fisher, F. Wilczek, and T. M. Yu, "Observability of earth-skimming ultra-high energy neutrinos," Phys. Rev. Lett., vol. 88, p. 161102, 2002. e-print: hep-ph/0105067

[18] D. Fargion, A. Aiello, and R. Conversano, "Horizontal tau air showers from mountains in deep valley: Traces of UHECR neutrino/tau," 1999. e-print: astro-ph/9906450

[19] F. Vannucci, "Detecting cosmic rays of the highest energies," in NATO Advanced Research Workshop on Cosmic Radation: From Astronomy to Particle Physics, Oujda, Morocco, Mar 21-23, 2001, submitted for publication.

[20] C. Hojvat, "The Pierre Auger observatory," presented at 32nd Rencontres de Moriond: High-Energy Phenomena in Astrophysics, Les Arcs, France, 18-25 Jan 1997.

[21] NuTel Collaboration. (2002) NuTel homepage. [Online]. Available: http://www.nutel.org/

[22] G. W. S. Hou and M. A. Huang, "Expected performance of a neutrino telescope for seeing AGN/GC behind a mountain," Nucl. Phys. Proc. Suppl., vol. 118, pp. 425-437, 2003. e-print: astro-ph/0204145

[23] M. H. Huang, J. J. Tseng, and G. L. Lin, in International Cosmic Ray Conference, Tsukuba, Japan, July 31 - August 7, 2003, submitted for publication.

[24] O. E. Kalashev, V. A. Kuzmin, D. V. Semikoz, and G. Sigl, "Ultra-high energy neutrino fluxes and their constraints," Phys. Rev., vol. D66, p. 063004, 2002. e-print: hep-ph/0205050

[25] G. Hermann, C. Kohler, T. Kutter, and W. Hofmann, "Triggering of imaging air Cerenkov telescopes: PMT trigger rates due to night sky photons," 1995. e-print: astro-ph/9508028
[26] S. Preuss, G. Hermann, W. Hofmann, and A. Kohnle, "Study of the photon flux from the night sky at La Palma and Namibia, in the wavelength region relevant for imaging atmospheric Cherenkov telescopes," Nucl. Instrum. Meth., vol. A481, pp. 229-240, 2002. e-print: astro-ph/0107120

[27] E. N. Hayes, "Smithsonian's satellite-tracking program ... [part 1]," in Annual Report for the Smithsonian Institution for the Year ... 1961, 1961, p. 286.

[28] M. Sasaki, Y. Asaoka, and M. Jobashi, "Self-triggered image intensifier tube for high-resolution UHECR imaging detector," Nucl. Instrum. Meth., vol. A501, pp. 359-366, 2003.

[29] A. Go, "RICH detectors for the LHCb experiment," Nucl. Instrum. Meth., vol. A446, pp. 305-309, 2000.

[30] D. Websdale, private communication, Imperial College, 2003.

[31] K. Y. Lo et al., "AMiBA: Array for microwave background anisotropy," 2000. e-print: astro-ph/0012282 